\title{
Neuropathic sensory symptoms did not accurately detect polyneuropathy in type 2 diabetes mellitus
}

Franse LV, Valk GD, Dekker JH, et al. "Numbness of the feet" is a poor indicator for polyneuropathy in type 2 diabetic patients. Diabet Med 2000 Feb;17:105-10.

QUESTION: How accurate are sensory symptoms for predicting polyneuropathy
detected by clinical neurological examination in patients with type 2 diabetes mellitus?

Design

Blinded comparison of a neuropathic sensory symptom questionnaire with a clinical neurological examination.

Setting

26 general practices in the Netherlands.

\section{Patients}

588 patients (mean age 67 y, 53\% women) with type 2 diabetes who received diabetes care in general practice. Patients were excluded if they received diabetes care in a hospital outpatient clinic or had other aetiological factors that might affect sensory functions of the skin of the lower extremities.

Description of test and diagnostic standard Patients completed a 34 item diabetes symptom checklist that measured the physical and psychological symptoms related to type 2 diabetes; 10 items related to neuropathic sensory symptoms. The diagnostic standard was a clinical neurological examination done by a trained research clinician who was blinded to the results of the checklist. The total score on the clinical neurological examination could range between 0 and 25 and was the sum of scores determined by sensory modalities, ankle reflexes, and the anatomic level below which light touch sense was impaired; a total score $>4$ implied a diagnosis of diabetic polyneuropathy.

\section{Main outcome measures}

Diagnostic test properties of a neuropathic sensory symptom questionnaire.

\section{Main results}

$32 \%$ of patients had diabetic polyneuropathy detected by the clinical neurological examination. In univariate regression analyses, neuropathic symptoms associated with the clinical neurological examination were numbness of the feet $(\beta=0.35, \mathrm{p}<0.001)$, sensory alteration $(\beta=0.27, p<0.001)$, and symptoms of pain $(\beta=0.16$, $\mathrm{p}<0.001)$. The table shows sensitivities, specificities, and likelihood ratios for daily symptoms of numbness of the feet in patients $<68$ and $\geqslant 68$ years of age.

For correspondence: Dr L Franse, Institute for Research in

Extramural Medicine, Vrije Universiteit, Van der Boechorststraat 7, 1081 BT Amsterdam, the Netherlands. Fax +31204448361 .

Test characteristics for daily symptoms of numbness of the feet for detecting diabetic polyneuropathy*

\begin{tabular}{lllll}
$\begin{array}{l}\text { Patient } \\
\text { age }\end{array}$ & $\begin{array}{l}\text { Sensitivity } \\
(95 \% \text { Cl }) \dagger\end{array}$ & $\begin{array}{l}\text { Specificity } \\
\text { (Cl) } \dagger\end{array}$ & +LR & -LR \\
\hline$<68$ y & $28 \%(13$ to 44$)$ & $93 \%(87$ to 97$)$ & 4.0 & 0.77 \\
\hline$\geqslant 68$ y & $22 \%(9$ to 37$)$ & $92 \%(85$ to 97$)$ & 2.75 & 0.85 \\
\hline
\end{tabular}

*Abbreviations defined in glossary; LRs calculated from data in article. †Cls provided by author.

\section{COMMENTARY}

Peripheral neuropathy is a common and troublesome complication of diabetes mellitus. It increases the risk for diabetic foot disease, which accounts for more hospital bed occupancy than any other specific diabetic complication. If neuropathy is detected, closer monitoring and educational and therapeutic interventions can delay or prevent ulcers and amputations. Unfortunately, many of the growing number of people with diabetes are not being screened regularly for neuropathy. This situation might be improved if quick and simple screening tests were readily available in primary care, where most patients are now routinely reviewed.

Screening tests should be evaluated against a recognised diagnostic standard in the population in which the test is to be used by researchers blinded to the diagnostic test results. In this study by Franse $e$ al, a symptom questionnaire was tested against a clinical examination that had been previously validated against neurophysiological examination, albeit not in a community sample. The results were disappointing. Although symptoms were associated with examination findings, sensitivities for detection of neuropathy were $<30 \%$, and the authors concluded that the questionnaire should not replace annual examination by the general practitioner. Other researchers have suggested that symptom scores may be useful when combined with a limited screening examination. ${ }^{1}$ However, as monofilaments for testing sensation are now widely available, inexpensive, and easily administered by any trained practitioner, ${ }^{2}$ perhaps the need for a questionnaire to stratify the population and target examination by general practitioners is diminished.

Simon Griffin, MBBS, MSc

University Forvie Site Cambridge, $U K$

1 Feldman EL, Stevens MJ, Thomas PK, et al. A practical two-step quantitative clinical and electrophysiological assessment for the diagnosis and staging of diabetic neuropathy Diabetes Care 1994;17:1281-9.

Conclusion

Neuropathic sensory symptoms did not accurately detect polyneuropathy in patients with type 2 diabetes mellitus. 